\title{
Vasculature Disruption Enhances Bacterial Targeting of Autochthonous Tumors
}

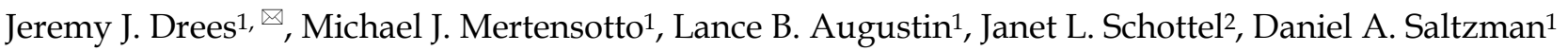 \\ 1. Department of Surgery, University of Minnesota, 195 MMC, 420 Delaware St SE, Minneapolis, Minnesota 55455, USA \\ 2. Department of Biochemistry, Molecular Biology \& Biophysics, University of Minnesota, 1479 Gortner Ave., St. Paul, MN 55108, USA
}

\author{
$\bowtie$ Corresponding author: Email: dree0036@umn.edu; Phone: 612-624-1994 \\ (C) 2015 Ivyspring International Publisher. Reproduction is permitted for personal, noncommercial use, provided that the article is in whole, unmodified, and properly cited. \\ See http://ivyspring.com/terms for terms and conditions.
}

Received: 2015.04.23; Accepted: 2015.06.23; Published: 2015.07.15

\begin{abstract}
Attenuated Salmonella enterica serovar Typhimurium (S. Typhimurium) has been developed as a vector to deliver therapeutic agents to tumors. The potential of $S$. Typhimurium in cancer therapy is largely due to its reported propensity to accumulate at greater than 1,000-fold higher concentrations in tumors relative to healthy tissues. In this study, we compared bacterial colonization of tumors in a subcutaneous transplantation model with a more clinically relevant autochthonous tumor model. Following intravenous administration of attenuated S. Typhimurium strain SL3261, we observed approximately 10,000-fold less bacteria in autochthonous tumors that sporadically develop in transgenic BALB-neuT mice compared to tumors developed from subcutaneous transplantation of $4 \mathrm{~T} 1$ murine breast cancer cells in BALB/c mice. Treatment of BALB-neuT mice with a vasculature-disrupting agent (VDA) prior to bacterial treatment caused necrosis of tumor tissue and significantly increased the bacterial targeting of autochthonous tumors by approximately 1,000 -fold. These observations emphasize the importance of appropriate model selection in developing bacteria-based cancer therapies and demonstrate the potential of combining VDA pre-treatment with bacteria to facilitate targeting of clinically relevant tumors.
\end{abstract}

Key words: Salmonella, cancer therapy, tumor-targeting, breast cancer, BALB-neuT, autochthonous, spontaneous, 4T1, vasculature disruption, necrosis, combretastatin A-4

\section{Introduction}

Despite over 40 years of fighting a declared "war on cancer," nearly 600,000 cancer-related deaths will occur in the United States this year and there will be over 8 million cancer-related deaths worldwide [1, 2]. Recent advances in cancer therapy demonstrate exciting potential, but are limited by toxic effects of systemically disseminated drugs [3]. In light of this, there is a growing interest in utilizing bacteria for cancer treatment due to the reported tumor-targeting propensity of microbes such as L. monocytogenes and S. enterica serovar Typhimurium (S. Typhimurium) [4]. Many published reports demonstrate the efficacy of attenuated $S$. Typhimurium strains in mouse models of cancer, either by themselves or as vectors to deliver recombinant therapeutic proteins directly to the tu- mor microenvironment [5]. In addition to efficacy, these studies report reproducibly high tumor colonization numbers of approximately $10^{9}$ colony-forming units (cfu) of $S$. Typhimurium per gram of tumor tissue with ratios of over 1000:1 when compared to healthy tissue [6].

When tested clinically, the results were largely disappointing. This was most likely due to a lack of tumor colonization. When tumors from 32 patients in clinical trials of parenterally administered $S$. Typhimurium strain VNP20009 were biopsied, less than $20 \%$ contained any measurable $S$. Typhimurium colonization [7-9]. The results of the VNP20009 trials were unexpected given the preclinical demonstrations of bacterial colonization of subcutaneous tumors. 
Since all of the preclinical studies were performed in transplant mouse models of cancer, it may be that the tumor-targeting observed in these models is due, at least partially, to characteristics of transplanted tumors that do not accurately mimic the autochthonous tumors that develop in human cancer. Necrotic tissue and hemorrhage of tumor blood vessels have been demonstrated to be important for the colonization of murine tumors [10, 11]. Moreover, both necrosis and immature vasculature have been shown to be present at artificially high levels in transplant models. Indeed, transplantation tumors often contain large necrotic regions due to rapid growth of the inoculated tumor cells, inflammation, and lack of mature vasculature, whereas human tumors develop much more slowly from a single cell and tend to be less necrotic due to suppression of an inflammatory immune response and coincident development of vasculature [12-14]. These observations may help explain the difference between the high bacterial colonization of tumors in transplantation mouse models and the low colonization observed in clinical studies.

The BALB-neuT model is a genetically engineered mouse model in which mammary tumor development is driven by expression of a constitutively activated rat homolog of human epidermal growth factor receptor 2 [15]. In this model, autochthonous tumors develop over several months and are palpable in the mammary pads of female mice around 16 weeks of age. We have recently reported efficacy with attenuated $S$. Typhimurium in the BALB-neuT model [16]. Yet, similar to results reported in human trials, no bacteria were observed in tumor tissue of treated mice at the time point tested, and the reduction in tumor burden was concluded to be due to a systemic immune response. To address bacterial targeting of different tumor types, we compared colonization of the transplantation tumors that develop in BALB/c mice subcutaneously inoculated with the syngeneic 4T1 breast cancer cell line to colonization of the autochthonous tumors that develop sporadically in the mammary glands of BALB-neuT mice. Since the vascular-disrupting agent (VDA) combretastatin A-4 phosphate (CA4P) has been used to enhance targeting of bacterial spores to transplanted tumors [17], we also investigated the use of CA4P to improve $S$. Typhimurium targeting of autochthonous tumors.

\section{Materials and methods}

\section{S. Typhimurium strain growth and prepara- tion for treatment:}

S. Typhimurium strains VNP20009 ( $\Delta m s b, \Delta p u r l)$ [18] and SL3261 ( $\triangle a r o A, \Delta h i s G)$ [19] were obtained from The American Type Culture Collection (ATCC
\#202165) and The Salmonella Genetic Stock Centre (SGSC \#439), respectively. Cultures of VNP20009 and SL3261 were grown from single colonies in lysogeny broth (LB) and MSB broth [20], respectively, at $37^{\circ} \mathrm{C}$ until reaching mid log phase at approximately $10^{8}$ $\mathrm{cfu} / \mathrm{ml}$. Cultures were then chilled by swirling the flasks in ice water, and diluted in ice-cold PBS to the concentrations required for injections.

\section{Mouse maintenance}

All mice were maintained in specific pathogen free conditions and fed standard mouse chow (Harlan). Research Animal Resources at the University of Minnesota provided animal care. All protocols were approved by the Institutional Animal Care and Use Committee.

\section{T1 experiments}

4T1 cells (ATCC \#CRL-2539) were grown in RPMI-1640 medium containing 10\% fetal bovine serum. Cells were detached from flasks using 0.05\% trypsin-EDTA, counted with a hemocytometer, washed with sterile PBS, and diluted to the appropriate concentration prior to injection. Female BALB/c mice of approximately 12 weeks of age were given a subcutaneous injection of $1 \times 10^{5} 4 \mathrm{~T} 1$ cells in $50 \mu \mathrm{L}$ of sterile PBS superficial to the right inguinal mammary fat pad. Tumor growth was measured over time by caliper. Mice were treated with $S$. Typhimurium by intravenous (IV) injection when tumors reached approximately $400 \mathrm{~mm}^{3}$ volume calculated as an ellipsoid (length $\times$ width $\times$ depth $\times 0.52$ ).

\section{BALB-neuT experiments}

BALB-neuT colony maintenance and husbandry was performed as previously described [16]. Tumor growth was monitored over time. Mice were treated by IV injection with $S$. Typhimurium when a single tumor reached $400 \mathrm{~mm}^{3}$. Most BALB-neuT mice had additional smaller tumors on other mammary pads; these tumors were not analyzed in this study.

\section{Treatment of mice, tissue harvest, and analysis}

Mice were injected IV with $5 \times 10^{5} \mathrm{cfu}$ of the appropriate $S$. Typhimurium strain in $100 \mu \mathrm{L}$ of PBS via the tail vein. For VDA pre-treatment, mice were given two IV injections of $0.4 \mathrm{mg}(20 \mathrm{mg} / \mathrm{kg})$ of combretastatin A-4 phosphate (CA4P): one injection 48 hours prior to and one injection immediately before $S$. Typhimurium treatment. Mice were euthanized 48 hours post-S. Typhimurium administration. Whole tumors and spleens were harvested, weighed, and minced with sterile razor blades to about $1 \mathrm{~mm}$ diameter pieces. The entire minced samples were then added to $3 \mathrm{ml}$ of sterile PBS and homogenized using gentleMACS M Tubes (Miltenyi Biotec) with a gen- 
tleMACS Tissue Dissociator (program RNA_01). The homogenate was serially diluted in sterile PBS, and $100 \mu \mathrm{l}$ of each dilution was plated on LB agar. Plates were incubated for $16-18$ hours at $37^{\circ} \mathrm{C}$ to allow colony formation. Colonies were then counted and colony-forming units/gram of tissue were calculated as the number of colonies per $0.1 \mathrm{~mL}$ plated $\times 10 \times$ total $\mathrm{mL}$ of sample (PBS + homogenized tissue) divided by tumor weight in grams. For histology, tumors were harvested from untreated control mice and from mice 48 hours after administration of $0.4 \mathrm{mg}$ CA4P. Tissue was fixed in 10\% neutral-buffered formalin solution for two days followed by $70 \%$ ethanol for two days. Tissues were then paraffin-embedded, sectioned and stained with hematoxylin and eosin (H\&E) according to standard protocols.

\section{Results}

\section{Attenuated S. Typhimurium targets subcuta- neous transplant but not autochthonous tu- mors}

Reports of cancer treatment using the attenuated S. Typhimurium strain VNP20009 demonstrated successful targeting of transplanted mouse tumors with IV injections. In a phase I clinical trial, the maximum tolerable dose of IV VNP20009 in human cancer patients was $3 \times 10^{8} \mathrm{cfu} / \mathrm{m}^{2}$ [21]. This dose translates to $2 \times 10^{6} \mathrm{cfu}$ per $20 \mathrm{~g}$ mouse. In order to avoid toxic effects, we used a dose of $5 \times 10^{5} \mathrm{cfu}$. IV administration of this dose to mice did not result in significant weight loss compared to saline-treated controls (data not shown). To verify successful tumor-targeting in a transplant model of breast cancer, 4T1 cells were injected subcutaneously superficial to the mammary fat pads of female BALB/c mice. When tumors reached $400 \mathrm{~mm}^{3}$ in volume, mice were given a single IV injection of VNP20009. Mice were harvested 48 hours post injection, and their tissues were examined for bacterial colonization. In agreement with published studies [5], we observed invariably high tumor colonization of greater than $1 \times 10^{8} \mathrm{cfu} /$ gram of tumor tissue, which was more than 1000 -fold greater than colonization of the spleen (Figure 1A). To investigate the colonization of autochthonous tumors, BALB-neuT mice with size-matched mammary tumors were given the same injection of VNP20009 and examined 48 hours later for tumor colonization. Compared to 4T1 tumors, the autochthonous BALB-neuT tumors contained approximately 10,000-fold fewer cfu/g (Figure 1A). While tumor colonization was greatly reduced in the autochthonous tumors, splenic colonization was similar when compared to mice bearing 4T1 tumors (Figure 1A).
It has been suggested that the failure of VNP20009 to successfully target human tumors may be due to its lack of lipopolysaccharide endotoxin, which may elicit a cytokine-dependent blood influx that is critical for tumor colonization [11, 22]. To address whether a Lipid $\mathrm{A}^{+}$strain would result in better BALB-neuT tumor colonization, we treated both 4T1 and BALB-neuT tumor-bearing mice as before using the attenuated $S$. Typhimurium strain SL3261. As observed with VNP20009, SL3261 readily colonized 4T1 tumors, but not autochthonous BALB-neuT tumors (Figure 1B). In attempts to improve tumor colonization, we tested doses of SL3261 and VNP20009 containing up to $5 \times 10^{6} \mathrm{cfu}$ and also included "primer" doses of varying sizes, which have been reported to improve tumor-targeting in some instances [23]. While increasing colonization marginally, these dosing strategies were limited by toxicity, as mice that received $2 \times 10^{6}-3 \times 10^{6}$ total cfu of either strain experienced significant weight loss, and doses $>3 \times 10^{6} \mathrm{cfu}$ were lethal in some cases (data not shown).

\section{Vascular disruption improves tumor-targeting of autochthonous mammary tumors}

As an alternative approach to improve tumor-targeting, we investigated whether pre-treatment of mice with a vasculature-disrupting agent (VDA) would make autochthonous tumors more susceptible to colonization by affecting vasculature and necrosis. VDAs cause the destruction of immature vasculature that is often found in tumor tissue, which leads to hemorrhagic necrosis [24]. Female BALB-neuT mice with autochthonous mammary tumors were given two IV injections of the VDA CA4P at $20 \mathrm{mg} / \mathrm{kg}$, 48 hours apart. Immediately following the second CA4P injection, mice were injected IV with $5 \times 10^{5}$ cfu of SL3261 and harvested two days later to determine tissue colonization. When SL3261 injection was combined with CA4P pre-treatment, there was an approximate 1000 -fold increase in tumor colonization when compared to mice not pre-treated with CA4P, while splenic colonization was not affected (Figure 1B). The combination of CA4P pre-treatment with SL3261 treatment did not cause any overt behavioral changes or significant weight loss of the mice.

To verify the effects of CA4P pre-treatment on tumor tissue, tumors from BALB-neuT mice treated with CA4P were excised two days post-treatment and histologically compared to tumors from untreated mice. As expected, CA4P treatment induced the formation of necrotic regions that were largely absent in the tumors of untreated mice (Figure 2). 


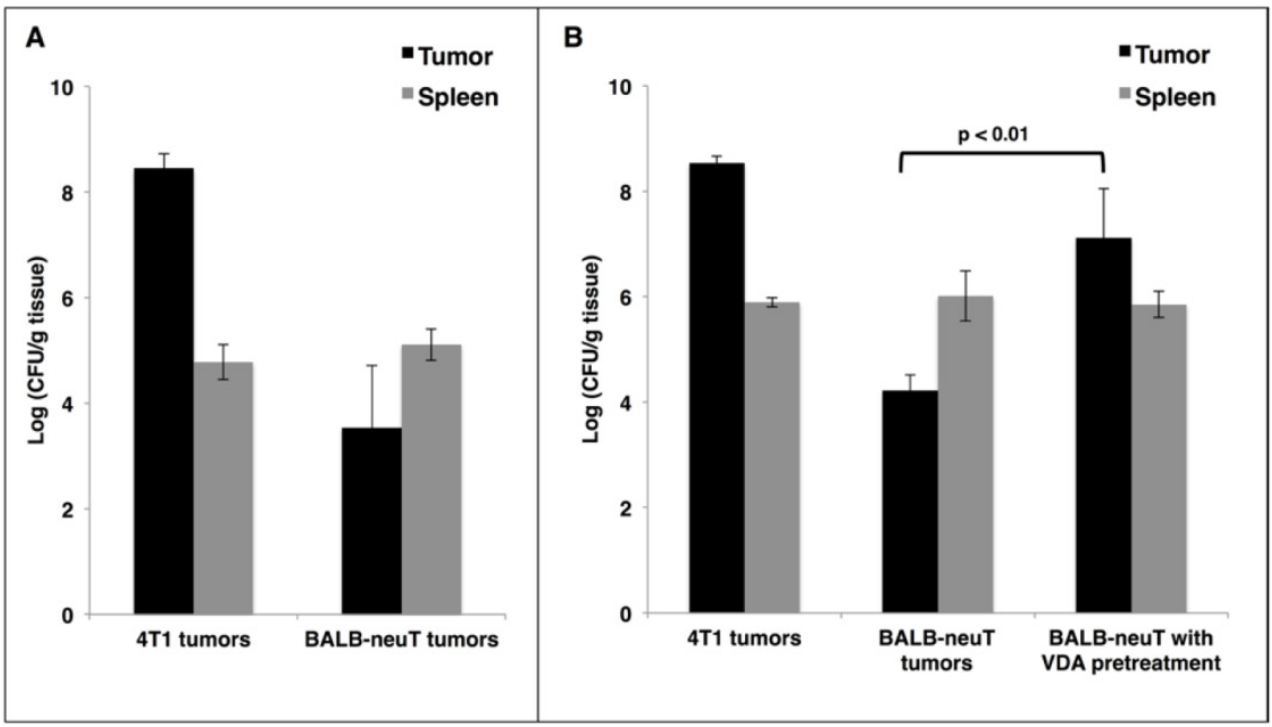

Figure 1: VDA improves tumor-targeting of attenuated S. Typhimurium in autochthonous breast cancer. A: BALB/c mice bearing subcutaneous 4T1 breast tumors or BALB-neuT mice bearing size-matched sporadic tumors were administered $5 \times 10^{5}$ cfu of log phase VNP20009 by tail vein injection. Tumors and spleens were harvested from mice two days post bacterial injection and were homogenized and plated for cfu determination. B: Mice bearing 4T1 or BALB-neuT tumors were treated as in (A) with log phase SL3261 and similarly plated for cfu determination. The VDA pre-treatment group was administered $0.4 \mathrm{mg}$ CA4P 48 hours prior to, and once again immediately before, injection of SL3261. For panels $A$ and B, the $\log _{10}$ of individual cfu/g values were used to calculate average and standard deviation of each group. Displayed is the average +/- one standard deviation of two separate experiments. Total mice for either the VNP20009 or SL3261 experiments: 4T1 N = 4; BALB-neuT N $=6$.

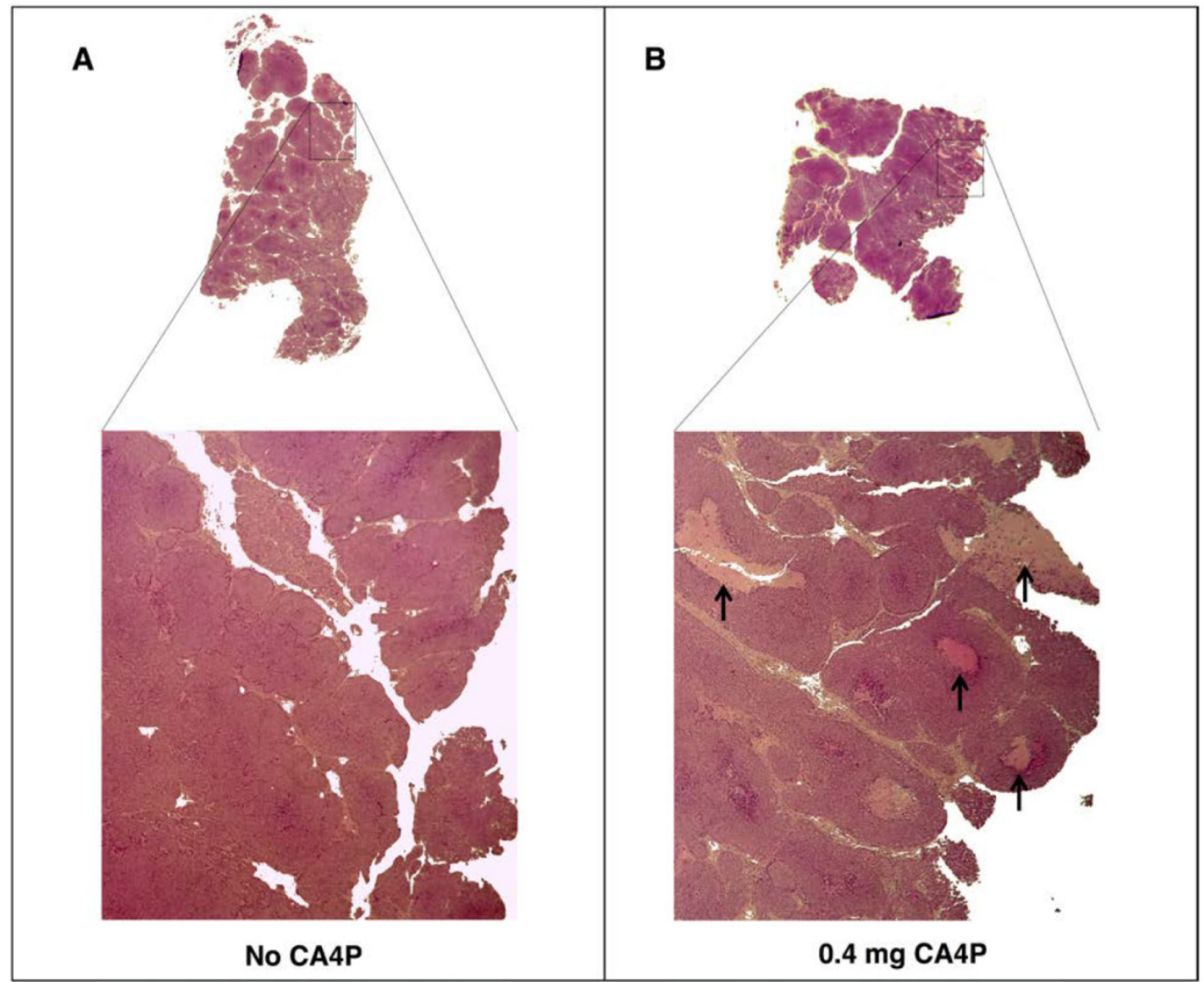

Figure 2: VDA treatment leads to development of necrotic areas in autochthonous BALB-neuT tumors. BALB-neuT mice with autochthonous mammary tumors were administered a single tail vein injection of $0.4 \mathrm{mg}$ of CA4P. Tumors were excised two days later for slide preparation and H\&E staining. (A) Necrotic regions were largely absent from CA4P (-) control tumors. (B) CA4P treatment caused the development of necrotic regions, indicated by black arrows. 


\section{Discussion}

The use of bacteria as nontoxic cancer therapy is based on the ability of virulence-attenuated bacterial strains to specifically target tumor tissue. This characteristic is highly reproducible in murine tumors developed from transplanted cells or tissues [5]. In the current study, however, we observed that the more clinically relevant autochthonous BALB-neuT tumors are much less readily colonized by IV-administered bacteria than are transplanted subcutaneous $4 \mathrm{~T} 1 \mathrm{tu}-$ mors. These results are consistent with $S$. Typhimurium failing to colonize tumors when tested in a clinical setting and may explain the lack of significant efficacy observed in the clinical trials [7-9]. Therefore, it is important for future preclinical studies of tumor colonization to utilize autochthonous models that more closely mimic the development of human tumors.

Recently reported studies suggest that $S$. Typhimurium colonization of tumors is constrained by both the amount of necrotic space and the capacity of tumor vasculature to facilitate bacterial extravasation $[10,11,25]$. Because combretastatin A-4 causes hemorrhagic necrosis by breaking down the epithelial wall of immature vasculature in tumors [24], we tested its ability to enhance colonization of autochthonous tumors by IV administered S. Typhimurium. The observed 1000-fold average increase in colonization with CA4P pre-treatment confirmed the ability of this VDA to enhance tumor colonization. While CA4P increased necrotic regions in the tumors of BALB-neuT mice, it is not clear if extravasation of bacteria from vasculature, establishment of necrotic regions, or both contributed to the colonization of BALB-neuT tumors. However, in preliminary optimization experiments (not shown), we found that the timing of the two CA4P doses was important, and a single dose of CA4P was ineffective at improving tumor colonization. The dosing strategy described in this report that included two doses 48 hours apart followed immediately by bacterial injection was optimal. We suspect that the first dose causes the development of necrotic regions important for tumor colonization (Figure 2), and the second injection disrupts the nascent vasculature that begins to form after the first injection, causing hemorrhage of the tumor blood vessels and facilitating entry of bacteria into the tumor. Experimentation to optimize the timing and concentration of VDA dosing may clarify the relative contribution of each mechanism to improved bacterial colonization. Continued investigation of how autochthonous tumors can be conditioned to increase targeting by bacteria will increase the likelihood of successful clinical translation of bacterial cancer therapy.

\section{Acknowledgments}

Funding was provided by ProjectStealth.org, StoneArch, the Randy Shaver Cancer Research and Community Fund, the Department of Surgery at the University of Minnesota, and the ASL Cancer Research Fund. The authors also would like to thank the Minnesota Supercomputing Institute for providing necessary software licenses for this research. A special thanks goes to Brenda Koniar (Research Animal Resources, University of Minnesota) for assistance with animal handling and husbandry; David Urso (Department of Surgery Histology Core, University of Minnesota) for tissue sectioning and H\&E staining; Jacob Zulk, Scott Durdin, Emily Balczewski, and Joseph Lane for assistance with the experiments; and Dr. Arnold Leonard for valuable discussion.

\section{Abbreviations}

S. Typhimurium: Salmonella enterica Typhimurium, VDA: vascular-disrupting agent, CA4P: combretastatin A-4 phosphate, cfu: colony-forming units, IV: intravenous.

\section{Competing Interests}

The authors have declared that no competing interest exists.

\section{References}

1. Hanahan D. Rethinking the war on cancer. Lancet. 2014; 383: 558-63.

2. Stewart BW, Wild C, International Agency for Research on Cancer, World Health Organization. World cancer report 2014. Lyon, France Geneva, Switzerland: International Agency for Research on Cancer WHO Press; 2014.

3. Alatrash G, Jakher H, Stafford PD, Mittendorf EA. Cancer immunotherapies, their safety and toxicity. Expert opinion on drug safety. 2013; 12: 631-45.

4. Paterson Y, Guirnalda PD, Wood LM. Listeria and Salmonella bacterial vectors of tumor-associated antigens for cancer immunotherapy. Semin Immunol. 2010; 22 : 183-9.

5. Forbes NS. Engineering the perfect (bacterial) cancer therapy. Nature reviews Cancer. 2010; 10: 785-94.

6. Pawelek JM, Low KB, Bermudes D. Tumor-targeted Salmonella as a novel anticancer vector. Cancer Res. 1997; 57: 4537-44.

7. Nemunaitis J, Cunningham C, Senzer N, Kuhn J, Cramm J, Litz C, et al. Pilot trial of genetically modified, attenuated Salmonella expressing the E. coli cytosine deaminase gene in refractory cancer patients. Cancer Gene Ther. 2003; 10: 737-44.

8. Toso JF, Gill VJ, Hwu P, Marincola FM, Restifo NP, Schwartzentruber DJ, et al. Phase I study of the intravenous administration of attenuated Salmonella typhimurium to patients with metastatic melanoma. J Clin Oncol. 2002; 20: 142-52.

9. Heimann DM, Rosenberg SA. Continuous intravenous administration of live genetically modified salmonella typhimurium in patients with metastatic melanoma. J Immunother. 2003; 26: 179-80.

10. Yu YA, Zhang Q, Szalay AA. Establishment and characterization of conditions required for tumor colonization by intravenously delivered bacteria. Biotechnol Bioeng. 2008; 100: 567-78

11. Leschner S, Westphal K, Dietrich N, Viegas N, Jablonska J, Lyszkiewicz M, et al. Tumor invasion of Salmonella enterica serovar Typhimurium is accompanied by strong hemorrhage promoted by TNF-alpha. PloS one. 2009; 4: e6692.

12. Falk P. Differences in vascular pattern between the spontaneous and the transplanted $\mathrm{C} 3 \mathrm{H}$ mouse mammary carcinoma. European journal of cancer \& clinical oncology. 1982; 18: 155-65

13. Lee JC, Kim DC, Gee MS, Saunders HM, Sehgal CM, Feldman MD, et al. Interleukin-12 inhibits angiogenesis and growth of transplanted but not in situ 
mouse mammary tumor virus-induced mammary carcinomas. Cancer Res. 2002; 62: 747-55

14. Schreiber K, Rowley DA, Riethmuller G, Schreiber H. Cancer immunotherapy and preclinical studies: why we are not wasting our time with animal experiments. Hematol Oncol Clin North Am. 2006; 20: 567-84.

15. Conti L, Ruiu R, Barutello G, Macagno M, Bandini S, Cavallo F, et al. Microenvironment, oncoantigens, and antitumor vaccination: lessons learned from BALB-neuT mice. BioMed research international. 2014; 2014: 534969.

16. Drees J, Mertensotto M, Liu G, Panyam J, Leonard A, Augustin L, et al. Attenuated Salmonella enterica Typhimurium Reduces Tumor Burden in an Autochthonous Breast Cancer Model. Anticancer Res. 2015; 35: 843-9.

17. Theys J, Landuyt W, Nuyts S, Van Mellaert L, Bosmans E, Rijnders A, et al. Improvement of Clostridium tumour targeting vectors evaluated in rat rhabdomyosarcomas. FEMS Immunol Med Microbiol. 2001; 30: 37-41.

18. Low KB, Ittensohn M, Le T, Platt J, Sodi S, Amoss M, et al. Lipid A mutant Salmonella with suppressed virulence and TNFalpha induction retain tumor-targeting in vivo. Nat Biotechnol. 1999; 17: 37-41.

19. Hoiseth SK, Stocker BA. Aromatic-dependent Salmonella typhimurium are non-virulent and effective as live vaccines. Nature. 1981; 291: 238-9.

20. Murray SR, Bermudes D, de Felipe KS, Low KB. Extragenic suppressors of growth defects in msbB Salmonella. J Bacteriol. 2001; 183: 5554-61.

21. Toso JF, Gill VJ, Hwu P, Marincola FM, Restifo NP, Schwartzentruber DJ, et al. Phase I study of the intravenous administration of attenuated Salmonella typhimurium to patients with metastatic melanoma. Journal of clinical oncology : official journal of the American Society of Clinical Oncology. 2002; 20: $142-52$.

22. Leschner S, Weiss S. Salmonella-allies in the fight against cancer. J Mol Med (Berl). 2010; 88: 763-73.

23. Tome Y, Zhang Y, Momiyama M, Maehara H, Kanaya F, Tomita K, et al. Primer dosing of S. typhimurium A1-R potentiates tumor-targeting and efficacy in immunocompetent mice. Anticancer Res. 2013; 33: 97-102.

24. Tozer GM, Kanthou C, Baguley BC. Disrupting tumour blood vessels. Nat Rev Cancer. 2005; 5: 423-35

25. Zhang M, Swofford CA, Forbes NS. Lipid A controls the robustness of intratumoral accumulation of attenuated Salmonella in mice. Int J Cancer. 2014; 135: 647-57. 